\title{
The Mathematical Model for Particle Suspension Flow through Porous Medium
}

\author{
Hooman Fallah, Hosein Barzegar Fathi, Hamed Mohammadi \\ Department of Petroleum Engineering, Firoozabad Branch, Islamic Azad University, Firoozabad, Iran \\ Email: hooman.fallah2@gmail.com
}

Received May 12, 2012; revised June 14, 2012; accepted June 24, 2012

\begin{abstract}
Transport of suspensions and emulsions in porous media occurs in numerous processes of environmental, chemical, petroleum and civil engineering. In this work, a mass balance particle transport equation which includes filtration has been developed. The steady-state transport equation is presented and the solution to the complete advective-dispersion equation for particulate suspension flow has been derived for the case of a constant filter coefficient. This model includes transport parameters which are particle advective velocity, particle longitudinal dispersion coefficient and filter coefficient. This work recommends to be investigated by particle longitudinal dispersion calculation from experimental data, directly. Besides, the numerical model needs to be developed for general case of a transition filter coefficient.
\end{abstract}

Keywords: Particulate Suspension Flow; Filtration Theory; Filtration Coefficient; Transport; Porous Medium; Mathematical Model

\section{Introduction}

The transport of particulate suspensions in porous medium occurs in a variety of industrial and natural process such as wastewater treatment, propagation of pollutants in subsurface environment, fouling of membranes and seawater injection in oil reservoirs. Flow of solid and liquid particles with particle capture by the rock takes place during injection of seawater and produced water in oil fields.

Particle transport and retention are mostly important for the environmental processes, where the particle concentration must not exceed a safety value, while the permeability changes is important for petroleum production due to its effect on well productivity and injectivity [1].

The study of particle retention in porous medium can be dated back to the work of Iwasaki [2] who proposed the following basic empirical for deep bed hydrosol filtration.

$$
\frac{\partial \sigma}{\partial t}=\lambda c u
$$

where: $\sigma$ is the specific volume of deposited material (particle retained concentration), $u$ is the fluid superficial velocity, $c$ is the particle suspended concentration and $\lambda$ is called the filtration coefficient.

The must used approach for evaluating colloid migration, retention and detachment is solute transport mass balance equation with the sink term for particle retention $[3,4]$.

$$
\begin{gathered}
\frac{\partial}{\partial t}(\phi c+\sigma)+U \frac{\partial c}{\partial x}=D_{o} \frac{\partial^{2} c}{\partial x^{2}} \\
\frac{\partial \sigma}{\partial t}=\lambda(\sigma) c U-k_{\mathrm{det}} \sigma
\end{gathered}
$$

The term $\lambda$ in Equation (3) is called filtration coefficient. Equations (1) and (2) together with the formula for coefficient $\lambda$ are called the classical filtration theory in above references.

In the current work, a theoretical investigation of particulate suspension flow through porous media has been done. The steady-state transport equation is presented and the solution has been derived. The filtration coefficient $\lambda$ will be discussed as a dominant parameter in the particle transport and retention through porous medium.

\section{Particulate Suspension Flow through Porous Medium}

\subsection{Filtration Theory}

When fluid containing particles reaches a porous medium, the liquid and the solid phase in the suspension can be separated, either by depositing in the pore or accumulating in front of the surface. This is similar to what happens in the filtration process. Then, the retention process of particles when flowing through a porous media is called Deep Bed Filtration [5]. The deep filtration occurs because of several mechanisms: the contacting of parti- 
cles with the retention site, the fixing of particle sites and the breaking away of previously retained particles [6].

\subsection{Mechanisms of Particles Retention}

The particle capture in porous media can be caused by different mechanisms [7].

- Size exclusion (large particles are captured in small pores and pass through large pores);

- Electrical forces (London-Van der Waals, double electrical layer, etc.);

- Gravity segregation;

- Multi particle bridging.

\section{Basic Formulation of Particles Transport through Porous Medium}

The model includes transport parameters which are particle advective velocity, particle longitudinal dispersion coefficient and filter coefficient. These parameters have been defined by dimensional analysis using the pertinent variables of the porous medium system.

\subsection{Advective Velocity}

The averaged particle velocity in the porous media, has been found to be either the same or slightly higher than that of the carrier fluid. This deviation is caused by the particle's size. The expected difference can be determined by analyzing the velocity profiles of both the fluid and the particles in a pore. The model has been formulated for a capillary tube which has a constant rate with the following assumption: No interactions between the particles and the wall, suspension is well-mixed with a constant concentration across the cross section .There is no transverse flow.

$$
\begin{aligned}
& U_{S}=U_{O}\left\{1-\left(\frac{r}{r_{o}}\right)^{2}\right\}\left(0<r<r_{0}\right) \\
& U_{P}=U_{O}\left\{1-\left\{\frac{r}{r_{o}}\right\}^{2}-\gamma\left\{\frac{a_{p}}{r_{o}}\right\}^{2}\right\}\left(0<r<r_{0}-a_{p}\right)
\end{aligned}
$$

As the particle travels through a tube, Brownian motion and shear action will cause the particle to travel across the entire cross-section of the tube except that the center-line of the particle will be excluded from the immediate region of the wall due to its radial dimension.

After the particle has traveled far enough longitudinally through the tube, the particle will have spent equal amounts of time in all radial position across the capillary tube. Integration of the velocity profile of Equation (4) over the range of possible radii shown in Equation (4) for both the particle and fluid yields the higher average velocity for the particle than that for the carrier fluid.

The average fluid velocity, $V_{S}$ in a capillary tube is:

$$
V_{S}=\frac{U_{O}}{2}
$$

The average velocity of a particle, $V_{P}$ in a capillary tube is:

$$
V_{P}=U_{O}\left(1-\frac{1}{2}\left\{1-\frac{a_{p}}{r_{o}}\right\}^{2}-\gamma\left\{\frac{a_{p}}{r_{o}}\right\}^{2}\right)
$$

By inspecting Equation (5) and (6), the particles are expected to have a larger average velocity than the carrier fluid velocity. This enhanced velocity of the particle can be expressed as a fractional difference between the two average velocities:

$$
\Delta V=\frac{V_{P}-V_{S}}{V_{S}}=2 \frac{a_{p}}{r_{o}}-\frac{7}{3}\left(\frac{a_{p}}{r_{o}}\right)^{2}
$$

This equation shows that as the radius of the particle increase, the difference between the average particle velocity and the average fluid interstitial velocity also increase. This increase is not unbounded but reaches a maximum $\Delta V$ value for $\frac{a_{p}}{r_{o}}=\frac{3}{7}$; as $\frac{a_{p}}{r_{o}}>\frac{3}{7}$ the velocity difference decrease. In physical sense, the pore radius can be estimated to approximately equal to one-fifth of media grain diameter $\left(r_{o}=\frac{1}{5} d_{g}\right)$; therefore the largest possible particle to be able to fit through the porous bed has a radius to this pore radius $\left(a_{p}=r_{o}\right)$.

For a particle with $a_{p}=r_{o}$, the particles have been shown to collect on the bed surface in a cake [6]. These references show that the onset of deep bed filtration occurs for a particle radius $a_{p}$.less than one-twentieth of the media grain diameter $\left(a_{p}<\frac{1}{20} d_{g}\right)$. Particles with the radii larger than this will not transport into the bed, but will collect on the surface. By lettingc $d_{g}=5 r_{o}$, the largest particle which will transport has a radius equal to one-fourth of the pore radius $\left(a_{p} \leq \frac{1}{4} r_{o}\right)$.

\subsection{Longitudinal Dispersion Coefficient}

An important element of any dispersion model is the representation of the geometry of the porous medium. Houseworth [8] has thoroughly reviewed such longitudinal dispersion model for solute tracers. Instead of modeling the internal structure of a porous medium, dimensional analysis is used to analyze the problem. In this study, the effect of mechanisms is expected to scale with the pertinent transport variables.

The pertinent variables for solute dispersion are:

$$
\begin{aligned}
& D_{L}=\text { longitudinal dispersion coefficient }\left(L \cdot T^{-1}\right) \\
& D=\text { free fluid molecular diffusion coefficient of }
\end{aligned}
$$




$$
\begin{aligned}
& \text { solute }\left(L^{2} \cdot T^{-1}\right) \\
\text { and } \quad V_{s}= & \text { fluid interstitial velocity }\left(L \cdot T^{-1}\right) \\
d_{g}= & \text { media grain diameter }(L)
\end{aligned}
$$

From the Buckingham pi theorem, the following pairs of groups are formed:

$$
\begin{gathered}
\frac{D_{L}}{V_{S} \cdot d_{g}}=F\left[\frac{V_{S} \cdot d_{g}}{D}\right], \text { oralternatively } \\
\frac{D_{L}}{D}=F\left[\frac{V_{S} \cdot d_{g}}{D}\right]
\end{gathered}
$$

where $P e=$ Pecletnumber $=\frac{V_{S} \cdot d_{g}}{D}$

Experimental data for solute longitudinal dispersion in uniform media show good correlation with these dimensionless groups [8]. When the Peclet number is grater than 1 , the two groups can be reduced to one:

$$
\frac{D_{L}}{V_{S} \cdot d_{g}} \approx \text { Constant }
$$

where $P e_{D}=$ dynamic Peclet number $=\frac{V_{S} \cdot d_{g}}{D_{L}}$

An order to magnitude approximation for the longitudinal dispersion coefficient for solutes can be made with:

$$
D_{L}=V_{S} \cdot d_{g}
$$

Particle longitudinal dispersion is expected to be similar to that of solutes. Currently, no particle breakthroughs have been performed by others form which particle longitudinal dispersion coefficient be determined.

\subsection{Filter Coefficient}

Two approaches exist for analyzing the filter coefficient. These are the macroscopic mass balance approach and the microscopic trajectory analysis approach. Deep bed filtration studies have been conducted to analyze both the system variables and the underlying mechanisms involved in the processes of capturing and retaining particles in porous media.

\subsubsection{Macroscopic Approach}

The macroscopic process of filtration or change in suspension concentration over depth is first-order decay with distance in steady flow. The filter coefficient may be expressed as a function of single collector efficiency and the single collector efficiency may be related to microscopic filtration mechanism.

Filtration results for steady state flow, neglecting longitudinal dispersion, gives:

$$
\frac{\partial C}{\partial x}=-\lambda C
$$

$$
C=C_{O} \cdot \exp [-\lambda x]
$$

The filter coefficient theoretically may be expressed as the single collector efficiency as follows:

$$
\lambda=\frac{3}{2} \frac{\left(1-\varnothing_{e}\right)}{d_{g}} \alpha_{c} \eta_{T}
$$

For $\eta_{T}$, the particles under consideration are those contained in a cylinder of diameter $d_{g}$ which is coincident with the vertical axis through the media grain collector. $\lambda$ can be found either experimentally or from the individual collector efficiency, $\eta_{T}$ and system variables using Equation (12).

The classical filtration theory assumes simultaneous particle capture and dislodging. On the contrary, the proposed model assumes that the particle capture takes place only if the total of torques for electrostatic and gravity forces prevails over the drag and lifting forces, so the resulting torque presses the particle towards the matrix or the internal cake.

\subsubsection{Microscopic Approach}

Microscopic study of particle motion near a collector is defined as trajectory analysis. In porous media, particle path far from media grains follow fluid streamline. As a particle approach a media grain, the motion deviate from the streamline because of various forces and torques acting on the particles. These forces are presented by transport and attachment mechanisms.

The transport mechanisms are: gravity settling, interception, Brownian diffusion and advection. The attachment mechanisms are considered to be gravity, London-van der Waals attraction, double layer forces.

From a combination of the trajectory analysis, the three major contribution of filtration can be formulated. These are collection due to Brownian diffusion, interception and settling. Here, we assumed double layer repulsive forces and hydrodynamic retardation (slow drainage of fluid from between two closely interacting particle which occurs before contact of particles) are negligible. The equations for these collection efficiencies are given in the following [7].

Collection due to Brownian diffusion,

$$
\eta_{D}=4 A_{S}^{\frac{1}{3}} \cdot P e_{p}^{-\frac{2}{3}}
$$

$4 A_{S}^{\frac{1}{3}}$ is constant. Also, either the particle Peclet number can be allowed to reach a minimum value of approximately 1 , or as $P e_{p}<1$ the efficiency reaches an asymptotic value of 1 .

Collection due to interception,

$$
\eta_{I}=1.5 \gamma^{2} A_{S} \cdot N_{R}^{2}
$$

$1.5 \gamma^{2}$ is constant here. Also the relative size group, $N_{R}$, can be allowed to reach a minimum value of ap- 
proximately 1 in order for the efficiency to remain less than or equal to 1 .

As we mentioned before, the size of the largest particles which are able to penetrate and transport through a porous bed is one twentieth of media grain diameter $\left(a_{p}<\frac{1}{20} d_{g}\right)$ or one-fourth of the pore radius $\left(a_{p}<\frac{1}{4} r_{o}\right)$. Collection due to settling,

$$
\eta_{G}=\frac{w_{s}}{V_{s}}=\frac{2\left(\rho_{p}-\rho_{f}\right) g a_{p}^{2}}{9 \mu V_{s}}
$$

The limit for this collection efficiency is the best possible collection which occurs for the settling velocity equaling the interstitial velocity. As the settling velocity becomes grater than the interstitial velocity, the efficiency remains at a value of 1 .

The equation for total collection efficiency is:

$$
\eta_{T}=\eta_{D}+\eta_{I}+\eta_{G}
$$
1.

In this case, $\eta_{T}$ has an asymptotic maximum value of

For Brownian particles ( $d_{p}<1.0$ micron $)$, there is good agreement between experimental and theoretical result but for advective particles ( $d_{p}<1.0$ micron $)$, it is not, because of neglecting hydrodynamic retardation and London-van der Waals attraction.

So, the exact analytical solution is [9]:

$$
\eta_{T}=4 A_{S}^{\frac{1}{3}} \cdot P e_{p}^{-\frac{2}{3}}+0.72 A_{S} N_{L O}^{\frac{1}{8}} N_{R}^{\frac{15}{8}}+2.4 E-3 A_{S} N_{G}^{\frac{1}{2}} N_{R}^{-\frac{4}{10}}
$$

The first term represents filtration due to Brownian diffusion. The second and third terms represent the combined effects of interception and gravity when the retardation and London-van der Waals attraction are included.

The effects of surface double layer forces are ignored (attractive surface double layer is controlled by transport processes and not depend on surface chemistry).

\section{Modeling of Particle Transport and Filtration}

In this part, the steady-state transport equation is presented and the solution to the complete advective-dispersion equation for particulate suspension flow has been derived for the case of a constant filter coefficient. This model includes transport parameters which are particle advestive velocity and particle longitudinal dispersion coefficient. These parameters have been defined by dimensional analysis using the pertinent variables of the porous media system.

\subsection{Particle Advective Velocity}

The result of the size exclusion for particles flowing in capillary tube can be written as Equation (6) where $\gamma=\frac{2}{3}$. By using the Equations (5), (6) and (7) we will have:

$$
\begin{aligned}
& V=1+\Delta V \\
& V^{*}=\frac{V_{P}}{V_{S}}
\end{aligned}
$$

As the particle size increase, the difference between particle velocity and fluid velocity increase.

\subsection{Particle Longitudinal Dispersion Coefficient}

In modeling particle dispersion, the following variable substitutions are used:

$$
\begin{aligned}
D & \rightarrow D_{P} \\
V & \rightarrow V_{P} \\
D_{L} & \rightarrow D_{L P}
\end{aligned}
$$

Particle size variable can be removed by using the particle properties as shown, provided $\frac{d_{p}}{d_{g}} \ll 1$ also the effect of particle size is included in the enhanced advective velocity for the particles.

This analysis shows that particle and solute longitudinal dispersion are similar. When the particle Peclet number $\left(P e_{p}=P e_{p}=\frac{V_{P} d_{g}}{D_{P}}\right.$ is grater than 10 , the two groups can be reduced to one :

$$
\frac{D_{L P}}{V_{P} d_{g}}=\text { constant }
$$

An order of magnitude approximation for the longitudinal dispersion coefficient for particles can be made with:

$$
D_{L P}=V_{P} d_{g}
$$

As mentioned before, the dimensional argument for defining the longitudinal dispersion coefficient is only valid when $P e_{p} \gg 1$. For uniform media, this restriction is seen to be $P e_{p}>10$. Flow conditions are simultaneously limited to the linear, laminar regime for which the Reynolds number must be less than 10 .

\subsection{Steady-State Transport Equation and Solution}

Particle removal or filtration occurs as a particle suspension flows through a porous medium due to the interaction of the advecting particles and grains of the medium. Iwasaki [1] is credited with being the first to express filtration a first-order decay of particle concentration with distance: 


$$
\begin{gathered}
\frac{\partial C}{\partial x}=-\lambda C \\
C(x=0)=C_{0}
\end{gathered}
$$

and a solution in dimensional terms:

$$
C=C_{0} \exp [-\lambda x]
$$

or in dimensionless terms:

$$
C^{*}=\exp \left[-\lambda^{*} x^{*}\right]
$$

where $C^{*}=\frac{C}{C_{0}}$

$$
\begin{gathered}
C_{0}=C(x=0) \\
\lambda^{*}=\lambda d_{g} \\
\mathrm{x}^{*}=\frac{\mathrm{x}}{\mathrm{d}_{\mathrm{g}}}
\end{gathered}
$$

A complete equation of steady-state filtration can be formulated by using the general steady-state advectiondispersion equation of transport for particle concentration with a sink term to describe particle removal due to filtration:

$$
0=D_{L P} \frac{\partial^{2} C}{\partial x^{2}}-V_{p} \frac{\partial C}{\partial x}-V_{p} \lambda C
$$

The following semi-infinite medium boundary conditions are:

$$
\begin{gathered}
C(x=0)=C_{0} \\
\lim _{x \rightarrow \infty} C(x)=0
\end{gathered}
$$

The solution which is shown in dimensional terms is derived in appendix:

$$
C(x)=C_{0} \exp [\alpha x]
$$

where $\alpha=\frac{1}{2} \frac{V_{P}}{D_{L P}}\left(1-\sqrt{1+4 \lambda \frac{D_{L P}}{V_{P}}}\right)$

$$
0=\frac{\partial^{2} C^{*}}{\partial x^{* 2}}-P e_{D p} \frac{\partial C^{*}}{\partial x^{*}}-P e_{D p} \lambda^{*} C^{*}
$$

With the same boundary conditions:

$$
\begin{gathered}
C^{*}\left(x^{*}=0\right)=1 \\
\lim _{x^{*} \rightarrow \infty} C^{*}\left(x^{*}\right)=0
\end{gathered}
$$

and a solution in dimensionless terms:

$$
\begin{gathered}
C^{*}=\exp \left[\alpha^{*} x^{*}\right] \\
\alpha^{*}=\alpha d_{g}=\frac{1}{2} P e_{D P}\left(1-\sqrt{1+\frac{4 \lambda^{*}}{P e_{D P}}}\right)
\end{gathered}
$$

\section{Conclusion}

In this work, a mass balance particle transport equation which includes filtration has been developed. This model includes transport parameters which are particle advective velocity, particle longitudinal dispersion coefficient and filter coefficient. The steady-state transport equation is presented and the solution to the complete advectivedispersion equation for particulate suspension flow has been derived for the case of a constant filter coefficient.

This work recommends to be investigated by particle longitudinal dispersion calculation from experimental data. Besides, the numerical model needs to be developed for general case of a transition filter coefficient.

\section{Acknowledgements}

The authors thank Professor P. Bedrikovetsky, Chair in Petroleum Engineering, Australian School of Petroleum, University of Adelaide.

\section{REFERENCES}

[1] S. Pang and M. M. Sharma, "A Model for Predicting Injectivity Decline in Water-Injection Wells,” SPE Paper 28489, Vol. 12, No. 3, 1997, pp. 194-201.

[2] T. Iwasaki, "Some Notes on Sand Filtration," Water Works Association, 1937, pp. 1591-1602,

[3] J. W. A. Foppen and J. F. Schijven, "Evaluation of Data from the Literature on the Transport and Survival of Escherichia coil in Aquifers under Saturated Conditions," Journal of Water Research, Vol. 40, No. 3, 2006, pp. 401-426. doi:10.1016/j.watres.2005.11.018

[4] J. F. Schijven and S. M. Hassanizadeh, "Removal of Viruses by Soil Passage: Overview of Modeling Processes, and Parameters," Critical Reviews in Environmental Science and Technology, Vol. 30, No. 1, 2000, pp. 49-127

[5] R. Farajzadeh, “An Experimental Investigation into Internal Filtration and External Cake Build Up,” MSc. Thesis, Delft University of Technology, 2004.

[6] J. P. Herzig, D. M. Leclerc and P. Le Goff, "Flow of suspension through porous media-application to deep filtration,” Journal of Industrial and Engineering Chemistry, Vol. 65, No. 5, 1970, pp. 8-35

[7] M. Elimelech and O'Melia, "Effect of Particle Size on Collosion Efficiency in the Deposition of Brownian Particles with Electrostatic Barriers,” 1990, pp. 1153-1163

[8] J. E. Houseworth, "Longitudinal Dispersion in Nonuniform, Isotropic Porous Media,” Ph.D. Thesis, W.M. Keck Laboratory of Hydraulics and Water Resources, California Institute of Technology, Report No. KH-R-45, 1984.

[9] A.C. Paytakes and C. Tien, "Particle Deposition in Fibrous Media with Dendritic Pattern: Apreliminary model,” Journal of Aerosol Science, Vol. 7, No. 2, 1976, pp. 85-94. doi:10.1016/0021-8502(76)90067-7 


\section{Appendix. Solution Derivation}

Consider the one-dimensional steady-state particle advective-dispersion equation which includes the removal term to account for filtration effects:

$$
0=D_{L P} \frac{\partial^{2} C}{\partial x^{2}}-V_{P} \frac{\partial C}{\partial x}-\lambda V_{P} C
$$

With the following boundary conditions:

$$
\begin{gathered}
C(x=0)=C_{0} \\
\lim _{x \rightarrow \infty} C(x)=0
\end{gathered}
$$

For convenience, the $\mathrm{x}$-variable is allowed to range from negative to positive infinity $(-\infty<x<\infty)$, although the equation are only applied for $x>0$ this avoids difficulty at $x=0$, because small dispersion is allowed. In dimensionless form, the transport equation becomes:

$$
0=D_{L P} \frac{\partial^{2} C^{*}}{\partial x^{* 2}}-P e_{D p} \frac{\partial C^{*}}{\partial x^{*}}-\lambda^{*} P e_{D p} C^{*}
$$

With the same boundary conditions:

$$
\begin{aligned}
& C^{*}\left(x^{*}=0\right)=1 \\
& \lim _{x^{*} \rightarrow \infty} C^{*}\left(x^{*}\right)=0
\end{aligned}
$$

In order to derive a solution, try the following as a solution:

$$
C^{*}\left(x^{*}\right)=\exp \left[\alpha^{*} x^{*}\right]
$$

Check the equation (A.3) by substituting into Equation (A.2), this results in second-degree polynomial in term of $\alpha^{*}$ and two roots of this polynomial are:

$$
\begin{aligned}
& \alpha_{1}^{*}=\frac{1}{2} P e_{D P}\left(1-\sqrt{1+\frac{4 \lambda^{*}}{P e_{D P}}}\right) \\
& \alpha_{1}^{*}=\frac{1}{2} P e_{D P}\left(1+\sqrt{1+\frac{4 \lambda^{*}}{P e_{D P}}}\right)
\end{aligned}
$$

Using these two roots, Equation (A.3) becomes:

$$
C^{*}\left(x^{*}\right)=A \exp \left[\alpha_{1}^{*} x^{*}\right]+B \exp \left[\alpha_{2}^{*} x^{*}\right]
$$

The constants of this Equation can be determined by applying the boundary conditions:

$$
\begin{gathered}
C^{*}\left(x^{*}=0\right)=1=A=B \\
C^{*}\left(x^{*} \rightarrow \infty\right)=0=(A)(0)+(B)(+\infty)
\end{gathered}
$$

$$
\therefore A=1 \text { and } B=0
$$

By substituting these constants into Equation (A.4), the solution to Equation (A.2) becomes:

$$
C^{*}\left(x^{*}\right)=\exp \left[\frac{1}{2} P e_{D P}\left(1-\sqrt{1+\frac{4 \lambda^{*}}{P e_{D P}}}\right) x^{*}\right]
$$

\section{Nomenclature}

$d_{g}=$ media grain diameter $(\mathrm{L})$

$d_{p}=$ particle diameter $(\mathrm{L})$

$c=$ suspended particle concentration in carrier fluid

$\sigma=$ particle retained concentration

$k_{\text {det }}=$ detachment rate coefficient

$U$ = flow velocity

$U_{S}=$ fluid velocity

$U_{P}=$ particle velocity

$U_{O}=$ fluid centerline velocity

$r=$ radial distance

$r_{o}=$ capillary radius

$a_{p}=$ particle radius

$p=$ dynamic pressure

$x=$ longitudinal distance

$D L=$ longitudinal dispersion coefficient $\left(\mathrm{L}^{\cdot} \mathrm{T}^{-1}\right)$

$D=$ free fluid molecular dispersion coefficient of solute

$\left(\mathrm{L}^{2} \cdot \mathrm{T}^{-1}\right)$

$V_{S}=$ fluid interstitial velocity $\left(\mathrm{L} \cdot \mathrm{T}^{-1}\right)$

$P e=$ Peclet number $=\frac{V_{S} \cdot d_{g}}{D L}$

$P e_{D}=$ dynamic Pec let number $=\frac{V_{S} \cdot d_{g}}{D L}$

$C=$ particle concentration $\left(\mathrm{M} \cdot \mathrm{L}^{-3}\right)$

$X=$ longitudinal position $(\mathrm{L})$

$\lambda=$ filter coefficient $\left(\mathrm{L}^{-1}\right)$

$W_{S}=$ particle settling velocity

$V_{S}=$ fluid interstitial velocity

$P_{p f}=$ densities of particle and fluid, respectively

$g=$ gravitational acceleration

$H=$ Hamakar constant (ergs)

$N_{G}=$ gravitional group $=\eta_{G}$

$D_{L P}=$ particle longitudinal dispersion coefficient $\left(\mathrm{L}^{2} \cdot \mathrm{T}^{-1}\right)$

$D P=$ particle molecular diffusion coefficient in a free fluid $\left(\mathrm{L}^{2} \cdot \mathrm{T}^{-1}\right)$

$V_{P}=$ particle velocity $\left(\mathrm{L}^{2} \cdot \mathrm{T}^{-1}\right)$ 